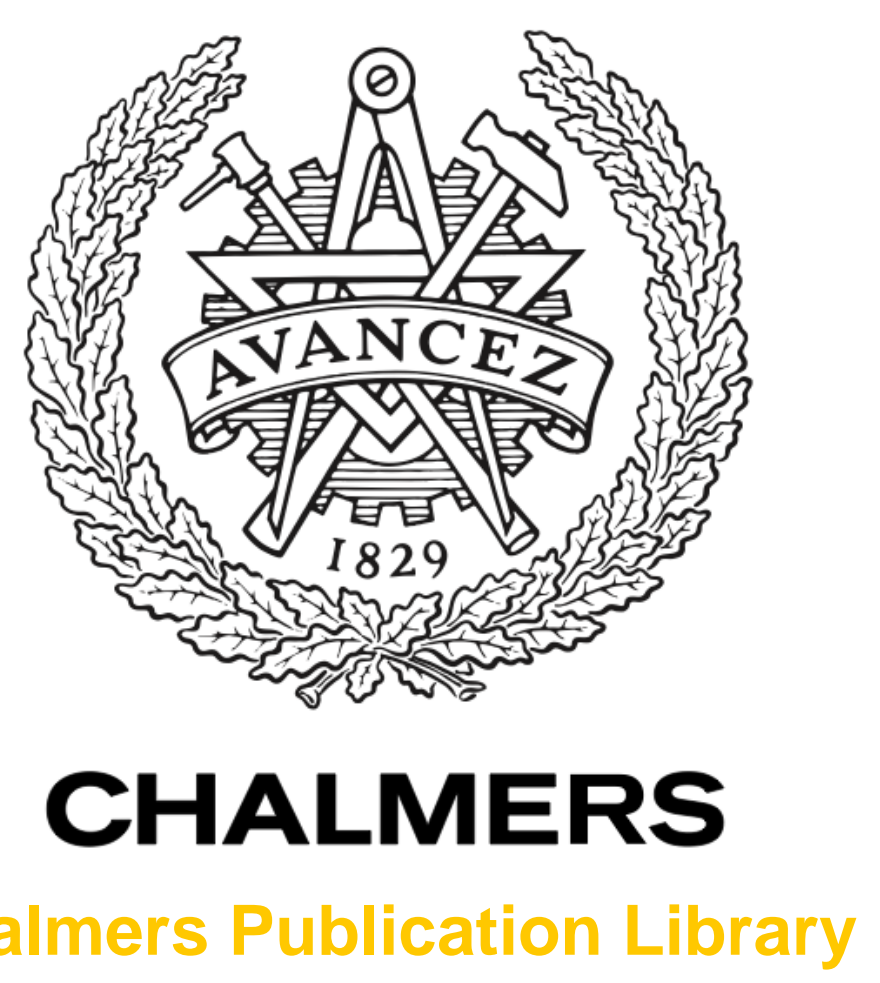

Chalmers Publication Library

Sustainable and robust Control of Cyclic Pallet Systems

This document has been downloaded from Chalmers Publication Library (CPL). It is the author's version of a work that was accepted for publication in:

2012 IEEE International Conference on Automation Science and Engineering: Green Automation Toward a Sustainable Society, CASE 2012, Seoul, 20-24 August 2012 (ISSN:

2161-8070)

Citation for the published paper:

Mashaei, M. ; Lennartson, B. (2012) "Sustainable and robust Control of Cyclic Pallet

Systems". 2012 IEEE International Conference on Automation Science and Engineering:

Green Automation Toward a Sustainable Society, CASE 2012, Seoul, 20-24 August 2012,

vol. 8 pp. 1184-1191.

http://dx.doi.org/10.1109/CoASE.2012.6386369

Downloaded from: http://publications.lib.chalmers.se/publication/171016

Notice: Changes introduced as a result of publishing processes such as copy-editing and formatting may not be reflected in this document. For a definitive version of this work, please refer to the published source. Please note that access to the published version might require a subscription.

Chalmers Publication Library (CPL) offers the possibility of retrieving research publications produced at Chalmers University of Technology. It covers all types of publications: articles, dissertations, licentiate theses, masters theses, conference papers, reports etc. Since 2006 it is the official tool for Chalmers official publication statistics. To ensure that Chalmers research results are disseminated as widely as possible, an Open Access Policy has been adopted.

The CPL service is administrated and maintained by Chalmers Library. 


\title{
Sustainable and robust Control of Cyclic Pallet Systems
}

\author{
Maziar Mashaei ${ }^{1,2}$, Bengt Lennartson ${ }^{1}$ \\ ${ }^{1}$ Department of Signals and Systems, Chalmers University of Technology, Sweden \\ ${ }^{2}$ FlexLink AB, Göteborg Sweden
}

\begin{abstract}
A proper control of a system to get a desired function and increase the system lifetime is a crucial step towards the sustainable paradigm. In this paper, such a control is designed for a cyclic pallet system to achieve a minimal force on its drive unit, meet safety conditions on the system chain tension force and the momentum of pallets, and fulfill the desired production rate. The optimal values of the control parameters, namely number of pallets and conveyor velocity, are obtained through solving a linear optimization model. The objective function in the model defines the average force on the drive unit in a cycle production. In addition, the related constraints characterize the pallet system properties such as cyclic and dynamic behavior, buffer size, constant work in process, and safety specifications. The robustness of the optimal control is analyzed, using a worst but safe control strategy. The optimal control and the robustness analysis are applied to some case studies, and the results are evaluated and discussed.
\end{abstract}

Key words: One-loop pallet system, Tension force model, Mixed integer linear programming, Optimal and robust control.

\section{INTRODUCTION}

Pallet systems are automated material handling mechanisms used in modern production environments to transport, buffer, and locate various part types. To facilitate the part handling and locating actions, the parts are loaded on standard carriers, pallets, which are equipped with Radio Frequency IDentification (RFID) tags. A conveyor chain transports the pallets to operating positions near to operating machines. When a pallet reaches to an operation position, the RFID tag in the pallet is fetched by a tag Reader/Writer, and the tag value is sent to a control system. Then, the pallet is fixated by a locating module if the control system specifies an operation for the part on the pallet. After the operation is finished, the pallet is released to follow its journey to the next operating position. At the last position in the handling path, the on board part is unloaded, and the empty pallet is directed to the loading section where a new part is loaded on the pallet. Accordingly, a closed path motion of pallets takes form.

Control of a one-loop pallet system has been the subject of interest for numerous studies. A paradigm shift from mass production to mass customization has reflected a variety of objectives in these studies. Researchers have devised models based on optimization frameworks [1], [2], queuing theory [3], [4], analytical methods [5], [6], [7], and discrete event formalism [8], [9], [10], [11], [12] to enhance the system performance [13], [14], [15], [16], minimize the makespan [17], [18], [19], and reduce the cycle time and work in process (WIP) [20], [21], [22], [23], [24]. These studies show the significant impact of the lean philosophy, [25], on control strategies of pallet systems. The time measurements and number of resources are the main indicators that express how effective are control policies, based on this philosophy.

Besides the mentioned indicators, there are other criteria for a pallet system which affect the performance and are highly focused in industries but not addressed in the research field. The sustainable design paradigm demands engineers in manufacturing sectors to enhance the lifetime of systems and products, [26]. For pallet systems, engineers in the design phase are concerned about two crucial criteria, which have significant impacts on the system lifetime. These are (a) the amount of the load which can be taken by the system without damaging the drive unit and (b) the number of pallets which are allowed to be queued behind a buffering device in a specific conveyor velocity. In the running phase, the more these criteria are violated, the more the system lifetime is decreased.

In practice, the evaluation of a control strategy for fulfillment of these constraints is a big challenge and demands a lot of time and effort in the acceptance test phase. Specially, it is more complicated and demanding if besides these constraints, the desired throughput should be satisfied as well.

In this paper, a new perspective on control of a one-loop pallet system is introduced to enhance the system lifetime and reduce the defects in the test phase, while the desired production rate is provided. Two indicators chain tension force and pallet momentum are developed in the phase of the control design, and the constraints which realize the desired throughput are addressed. It will be shown that such a control is obtained by proper tuning of the control parameters the number of pallets and the chain velocity through devising an optimization model. The robustness of the control strategy is furthermore discussed and characterized by using the concept of a worst but safe control.

\section{ASSUMPTIONS AND NotATIONS}

All mathematical models in this paper are developed based on the following assumptions and notations.

\section{Assumptions:}

- The pallet system has only one loading, unloading, and drive unit.

- Pallets are transported by the chain, and wheel type bends are used to change the orientation of a conveyor 
chain. This kind of bend nearly introduces zero friction between the chain and the bend slide.

- One pallet can handle only one product at a time, and the product remains clamped to the pallet during its entire journey in a pallet system.

- Operating times and transportation times are deterministic, and there is no failure in machines and the pallet system devices.

- The conveyor line transports pallets in a horizontal direction (there is no slope for conveyor lines).

- Empty pallets are reloaded with new parts as soon as possible. Besides, the empty pallet waiting times in the loading queue are negligible (As a matter of fact, this can also be assumed as a control objective).

- Universal (the same type of) pallets are used for different types of products.

- An assigned operation cannot be removed from a module until it is completed.

- A single resource can only be used for one operation at a time.

- All parts are removed when they reach the unloading section (no re-entrance).

- All locating modules follow the FIFO (first input first output) policy for a known schedule of the parts in the loading section.

- The chain is always running, and the acceleration time of a pallet from zero velocity to the chain velocity is negligible.

$\bullet$

\section{Notations:}

- $\ell \in M=\{1,2, \ldots, m\}$ is the index over locating modules. Moreover, $\ell=1$ and $\ell=m$ denote loading and unloading locating modules, respectively.

- $N^{\ell}(t)$ is the number of pallets at time $t$ in the segment including the module $\ell$. In addition, $N_{p}=\sum_{\ell=1}^{m} N^{\ell}(t)$ is the total number of pallets in the system.

- $L^{\ell}$ denotes the length of the segment related to the module $\ell$, and $b^{\ell}$ is the size of the buffer in this segment (including the locating space). Moreover, $L=\sum_{\ell=1}^{m} L^{\ell}$ is the overall length of the pallet system.

- $i \in I=\{1, \ldots, n\}$ is the index over parts and $\Pi=$ $\left\langle P_{1}, P_{2}, \ldots, P_{n}\right\rangle$ denotes an ordered part set.

- $\tau_{i}^{\ell}$ is the operating time in module $\ell$ for $P_{i}$.

- $v_{c}[\mathrm{~m} / \mathrm{s}]$ denotes the conveyor velocity, and $T_{c}=1 / v_{c}$ is the transportation time for one pallet passing 1 meter.

- $k \in K=\left\{1,2, \ldots k^{s}, k^{s}+1, \ldots, k_{U}^{s}\right\}$ is the index over cycles in transition and steady states. Moreover, $K^{s}=$ $\left\{k^{s}, k^{s}+1, \ldots, k_{U}^{s}\right\}$ includes all index cycles, in which the system is in the steady state phase.

- $S_{i}^{\ell}[k]$ is the time when $P_{i}$ is released by module $\ell$ in cycle $k$.

- $C_{i}^{\ell}[k]=S_{i+1}^{\ell}[k]-S_{i}^{\ell}[k]$ for $i \in I \backslash\{n\}$ and $C_{n}^{\ell}[k]=S_{1}^{\ell}[k+$ 1] $-S_{n}^{\ell}[k]$ are defined as interval times.

- $m_{c}$ is the mass of one meter chain $[\mathrm{kg} / \mathrm{m}]$ and $m_{p}$ is the average mass for a part (including its pallet) in the pallet system.
- $\mu_{s c}$ is the friction coefficient between the conveyor slide and conveyor chain, and $\mu_{c p}$ is the friction coefficient between the conveyor chain and a pallet.

- $\mathbb{N}, \mathbb{Z}$, and $\mathbb{R}$ denote the domain of variables for Natural $+\{0\}$, Integer, and Real numbers, respectively.

\section{ONE-LOOP PALLET SYSTEM}

A one-loop pallet system consists of a drive unit, a conveyor slide, a number of pallets, and a number of locating modules and buffering (stopping) devices. These equipments are defined as follows:

Conveyor slide: The conveyor slide is the main bone for a pallet system. The other devices in the system are connected or structured by the conveyor slide.

Drive unit: The drive unit includes a conveyor chain, an electrical motor, and related accessories (motor speed convertor, electrical driver, etc). The traction force caused by the electrical motor runs the conveyor chain in the conveyor slide.

Pallet: A pallet, which is transported by the conveyor chain with the friction force, carries a part (parts) towards operating positions. A universal pallet is equipped with an RFID tag identifying the on board part.

Locating module: A pallet should be located at right position before a related machine does any operation on its part. A locating module performs this action by disconnecting the pallet from the chain and fixating it at the position.

Buffering (Stopping) device: A stopping device can block the passage of pallets by an open-close gate and buffer them in a segment of the conveyor slide, while the chain is running under the pallets. This device is used to make a queue of pallets for a locating module.

One-loop pallet systems are often used in cyclic production lines with multiple part types. A production line is cyclic if it periodically produces an ordered set of parts $\left\langle P_{1}, P_{2}, \ldots, P_{n}\right\rangle$ in a specific duration denoted cycle time. One-loop pallet systems for cyclic production lines are from now called cyclic pallet systems. In a cyclic pallet system, although all parts pass the same order of modules from the loading to unloading section, their operating times and operation sequences can be different with respect to their types. Fig. 1 shows a cyclic pallet system with the set of parts $\left\{A, B_{1}, B_{2}\right\}$ and the locating modules $\left\{M^{1}, \ldots, M^{6}\right\}$. The sequence of operations for these parts are defined as follows:

- $A: M^{1} \rightarrow M^{2} \rightarrow M^{6}$

- $B_{1}: M^{1} \rightarrow M^{3} \rightarrow M^{5} \rightarrow M^{6}$

- $B_{2}: M^{1} \rightarrow M^{4} \rightarrow M^{5} \rightarrow M^{6}$

This means that for instance, part $B_{1}$ is loaded in $M^{1}$, located in the modules $\left\{M^{3}, M^{5}\right\}$ for processing operations, and unloaded in $M^{6}$.

\section{A. Challenges in control of a cyclic pallet system}

Gaining a desired value of the throughput is the main objective for control of a cyclic pallet system. According to [27] and [28], the average throughput $T H$ is related to 


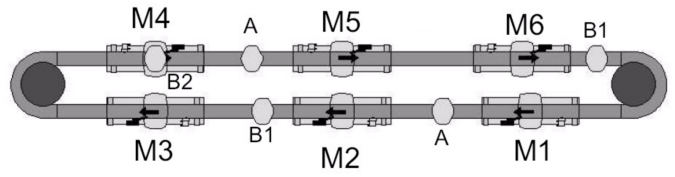

Fig. 1. One closed loop pallet system

the number of pallets $N_{p}$ and the average flow time $F T$, the duration in which a pallet runs the loop one time, by

$$
T H=\frac{N_{p}}{F T}
$$

Furthermore, $T H$ is calculated by the size of the part set $(n)$ divided by the cycle time $(C)$. Thus, (1) is modified to

$$
N_{p} C=n F T\left(N_{p}, v_{c}\right)
$$

in which the flow time is expressed as a function of the configurable parameters $N_{p}$ and the chain velocity $v_{c}$. In fact, a control problem of a cyclic pallet system demands a proper tuning of $N_{p}$ and $v_{c}$ to satisfy the equation (2) for the desired cycle time $C$. This is a great challenge because many dynamic and static parameters of the pallet system are involved in the adjustment of $F T$, which can not explicitly be formulated as a function of these parameters.

Besides the fulfillment of the desired throughput (cycle time), the other challenge is to safely run the pallet system without damaging its related resources. To make a realization of this control problem, we use data of an industrial pallet system.

X85 is one type of the pallet systems, which are designed and constructed by FlexLink company, [29]. To safely run $\mathrm{X} 85$, the designers should consider two crucial criteria. The first one puts a limitation on the momentum of pallets to be buffered behind a stopping device as illustrated in Fig. 2. In

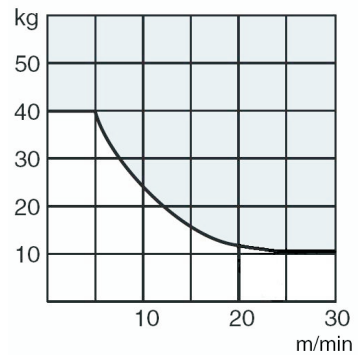

Fig. 2. Momentum is the product of mass and velocity. In this figure, the allowed momentum for a number of pallets buffered behind the stoping device is specified by the white color.

this figure, the safe domain is specified with the white color. For example, the stopping device can not take the average momentum higher than $3.33[\mathrm{~kg} \mathrm{~m} / \mathrm{s}]$. The second criterion introduces a constraint on the maximum tension force of the conveyor chain. The largest tension force, which varies in time, appears in the part of the chain connected to the shaft of the motor. Hence, it is crucial to bound this force lower than the maximum level which can be tolerated by the drive unit. Fig. 3 shows the maximum tension force versus the chain velocity and drive unit types for X85. For example, the tension force should not be higher than $800[\mathrm{~N}]$ for any velocity value provided that Medium drive unit is selected.

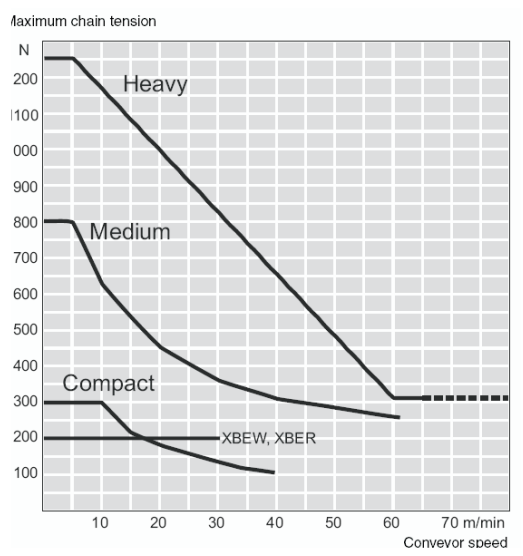

Fig. 3. Chain permissible tension force versus its velocity

These criteria on the momentum of pallets and the tension force can be generalized for any type of pallet systems. Hence, devising a control strategy to meet (2) and the safety conditions is a great challenge, but an important step towards the enhancement of the system lifetime and the sustainable paradigm. Furthermore, the strategy is more appreciated if the control parameters can be tuned such that the minimum force is applied on the drive unit. This means that a sustainable control of a cyclic pallet system can be addressed as follows.

Control problem: An optimal control of a cyclic pallet system is desired to have a minimal force on the drive unit, while the control fulfills the specified production rate and the criteria over the momentum and the tension force. The given parameters are system data, and the control parameters are $N_{p}$ and $v_{c}$.

\section{OPTIMIZATION MODEL OF THE CONTROL PROBLEM}

An optimization model of the control problem can be formulated as

$$
\begin{aligned}
& \min F\left(N_{p}, v_{c}\right) \\
& \text { subject to } \\
& \text { Design constraints }
\end{aligned}
$$

Here, $F\left(N_{p}, v_{c}\right)$ determines the average tension force as a function of $N_{p}$ and $v_{c}$, as well as the design constraints characterize (2) and the safety conditions. In [30], the authors have developed the mathematical models for the tension force and the design constraints without considering the safety conditions. In this section, these models are readdressed, and the novel constraints which characterize the safety conditions are introduced.

\section{A. Mathematical model for the mean value tension force}

A conveyor slide of a cyclic pallet system can be partitioned into $m$ segments such that each segment $\ell \in\{1, \ldots, m\}$ 
includes one locating module, one stopping device, and the part of the slide buffering pallets for the segment. According to [30], the largest tension force in the chain appears in the segment $\ell=m$ and obtained by

$$
\begin{aligned}
F^{m}(t) & =\left(N_{p} m_{p}+L m_{c}\right) g \mu_{s c}+m_{p} g \mu_{c p} \sum_{\ell=1}^{m} N_{s}^{\ell}(t) \\
& -m_{p} g\left(\mu_{s c}+\mu_{c p}\right) \sum_{\ell=1}^{m} N_{f}^{\ell}(t)
\end{aligned}
$$

where, $N_{s}^{\ell}(t)$ is the number of the pallets standing in the segment $\ell$ (not moving) at $t$,

$$
N_{f}^{\ell}(t)= \begin{cases}1 & \text { a pallet is located in the module } \ell \text { at } t \\ 0 & \text { a pallet is not located in the module } \ell \text { at } t\end{cases}
$$

and the other parameters have been defined in Section II. An upper band value of $F^{m}(t), F^{u}$, is estimated by setting $\sum_{\ell=1}^{m} N_{f}^{\ell}(t)=0$ and $\sum_{\ell=1}^{m} N_{s}^{\ell}(t)=N_{p}$. Accordingly,

$$
F^{u}=N_{p} m_{p} g\left(\mu_{s c}+\mu_{c p}\right)+L m_{c} g \mu_{s c}
$$

Denoting $\widetilde{F}\left(v_{c}\right)$ as the permissible tension force for the conveyor chain at $v_{c}$ (see Fig. 3), the condition $F^{u} \leq \widetilde{F}\left(v_{c}\right)$ guarantees a safe running of the drive unit. Furthermore, as derived in [30], the average tension force $F^{m}(t)$ in one cycle production leads to

$$
\begin{aligned}
F\left(N_{p}, T_{c}\right) & =m_{p} g\left(\mu_{s c}+\mu_{c p}\right) N_{p}-\frac{n L m_{p} g \mu_{c p}}{C} T_{c} \\
& +L m_{c} g \mu_{s c}-\frac{m_{p} g\left(\mu_{s c}+\mu_{c p}\right) \tau}{C}
\end{aligned}
$$

for $\tau=\sum_{i=1}^{n} \sum_{\ell=1}^{m} \tau_{i}^{\ell}$ and $T_{c}=1 / v_{c}$.

In (6), $N_{p}$ and $T_{c}$ can not take arbitrary values. To fulfill the cycle time $C$, these parameters should be set from the domains specified by the pallet system design constraints. In the next subsection, these constraints are introduced.

\section{B. Design constraint models}

A controlled pallet system should fulfill design properties categorized as control and plant constraints. The control constraints demand a proper coordination of the operations to satisfy the queuing and loading policies, desired cycle time, and safety specifications. The plant constraints, on the other hand, emphasize on the flow shop model of the production plant, closed queuing network form of the pallet system, and limited size of the buffers. These constraints are now modeled based on the assumptions and notations in Section II.

\section{1) Control constraints:}

Scheduling constraints: Let $\Delta_{n \times n}$ be a known permutation matrix in which its elements $[\Delta]_{i, j}=\delta_{i, j}$ satisfy the relations $\sum_{i=1}^{n} \delta_{i, j}=1$ and $\sum_{j=1}^{n} \delta_{i, j}=1$. This matrix characterizes a loading schedule of the ordered parts $\left\langle P_{1}, P_{2}, \ldots P_{n}\right\rangle$. Hence, based on FIFO policy, the scheduled operation time part $i$ in the module $\ell, T_{i}^{\ell}$, is calculated by

$$
\begin{aligned}
& i, j \in I, \quad \ell \in M \\
& \sum_{j=1}^{n} \delta_{i, j} \tau_{j}^{\ell}=T_{i}^{\ell}
\end{aligned}
$$

Cyclic and periodic constraints: These constraints demand two crucial properties for a controlled cyclic pallet system. The first and second constraints in (8) provide the periodic manner of releasing actions in a locating module $\left(C_{i}^{\ell}[k]=C_{i}^{\ell}\right)$. On the other hand, the last constraint, which forces these periodic actions to be performed in the exact duration of the cycle time, imposes the cyclic property of the releasing actions.

$$
\begin{array}{lll}
k \in K^{s}, \quad \ell \in M & \\
S_{i+1}^{\ell}[k]-S_{i}^{\ell}[k]=C_{i}^{\ell} \quad: \quad i \in I \backslash\{n\} \\
S_{1}^{\ell}[k+1]-S_{n}^{\ell}[k]=C_{n}^{\ell}: & k \in K^{s} \backslash\left\{k_{U}^{s}\right\} \\
\sum_{i=1}^{n} C_{i}^{\ell}=C &
\end{array}
$$

Safety constraints: The design conditions on the chain velocity, the chain tension force, and the pallets momentum define the safety constraints (9). The first constraint limits the velocity to be always lower than the maximum allowed velocity $\widetilde{V}$ for the system. The second one and the third one realize the condition $\widetilde{F}\left(v_{c}\right) \geq F^{u}$ discussed in (IV-A). In these constraints, $F^{u}$ is defined based on (5), and $\widetilde{F}\left(v_{c}\right)$ is approximated by $\min \left\{\widetilde{F} 1, \widetilde{F} 2, \widetilde{F} 2+\lambda\left(T_{c}-T\right)\right\}$, where $\widetilde{F} 1, \widetilde{F} 2, \lambda$, and $T$ are constant parameters. Except $\widetilde{F} 1$, the other parameters are estimated from the data given for the permissible tension force. For example, for Medium drive unit in Fig. 3 and $0 \leq v_{c} \leq 30 \frac{\mathrm{m}}{\mathrm{min}}, \widetilde{F} 2=800, \lambda=35$, and $T=12$. The parameter $\widetilde{F} 1$ is given according to the conveyor length.

$$
\begin{aligned}
& T_{c} \geq \frac{1}{\widetilde{V}} \\
& \min \{\widetilde{F} 1, \widetilde{F} 2\} \geq N_{p} m_{p} g\left(\mu_{s c}+\mu_{c p}\right)+L m_{c} g \mu_{s c} \\
& \widetilde{F} 2+\lambda\left(T_{c}-T\right) \geq N_{p} m_{p} g\left(\mu_{s c}+\mu_{c p}\right)+L m_{c} g \mu_{s c} \\
& b C \geq \sum_{i=1}^{n}\left(S_{i}^{\ell}[k]-S_{i}^{\ell-1}[k]\right) \quad: \quad \ell \in M \backslash\{1\}, k \in K^{s} \\
& \widetilde{I} T_{c} \geq(b-1) m_{p}
\end{aligned}
$$

Using the Little law [27], the forth constraint introduces the upper bound $b$ for the average number of pallets buffered in a segment (including a locating module). The last constraint, furthermore, limits the momentum of $(b-1)$ pallets standing in the buffer to be lower than $\widetilde{I}$, the permissible momentum for the stopping device (see Fig. 2).

\section{2) Plant constraints:}

Dynamic constraints: These constraints introduce a relation among the part release times $S_{i}^{\ell}[k]$, the transportation times $L^{\ell} T_{c}$, and the mapped operating times $T_{i}^{\ell}$. The open flow shop model of the production plant demands that each part goes to all processing machines with a unique order of the modules from the loading section to the unloading one. The first constraint in (10) characterizes such a dynamic behavior by neglecting the dynamic behavior of empty pallets. The rest of the constraints indicate the fact that $C_{i}^{\ell}[k] \geq T_{i}^{\ell}$, 
due to blocking and starving times.

$$
\begin{array}{lll}
i \in I, \quad k \in K, \quad \ell \in M & \\
S_{i}^{\ell}[k]-S_{i}^{\ell-1}[k] \geq T_{i}^{\ell}+L^{\ell} T_{c} & : \quad \ell \in M \backslash\{1\} \\
S_{i}^{\ell}[k]-S_{i-1}^{\ell}[k] \geq T_{i}^{\ell} & : & i \in I \backslash\{1\} \\
S_{1}^{\ell}[k+1]-S_{n}^{\ell}[k] \geq T_{1}^{\ell} & : & k \in K \backslash\left\{k_{U}^{s}\right\} \\
S_{1}^{1}[1] \geq T_{1}^{1} & &
\end{array}
$$

Definition (Starving and Blocking times): Starving time is the duration in which a module is in an idle state before receiving a pallet. The blocking time is the duration in which a pallet is blocked by a module (the operation has been finished, but the pallet is still located) due to the lack of space in the downstream buffer.

Pallet constraints: The following proposition introduces a relation between the number of pallets $N_{P}$, unloading interval times $C_{i}^{m}$, and flow time of $P_{i}, F T_{i}=S_{i}^{m}[k]-S_{i}^{1}[k]+$ $T_{i}^{1}+L^{1} T_{c}$ for the steady state phase $\left(k \in K^{s}\right)$.

Proposition (Pallet constraint): In a cyclic pallet system $F T_{i}$ for $i \in I=\{1, \ldots, n\}$ is obtained by

$$
\sum_{e=1}^{N_{p}} C_{g(i, e)}^{m}=S_{i}^{m}[k]-S_{i}^{1}[k]+T_{i}^{1}+L^{1} T_{c}: \quad i \in I
$$

where

$$
g(i, e)= \begin{cases}n: & i-e=n k, k \in \mathbb{Z} \\ (i-e) \quad \bmod n: & i-e \neq n k, k \in \mathbb{Z}\end{cases}
$$

Proof: Let $P_{x_{i}}$ be the part, which is now being unloaded, and the empty pallet of this part is loaded by $P_{i}$. Because all modules locate and release the parts according to FIFO policy, any unloading order of the part set is the same as the loading order of this set. Hence, the relation between indices of $P_{i}$ and $P_{x_{i}}$ can be shown by

$$
i=\left(x_{i}+N_{p}\right) \bmod n
$$

Now we observe that $F T_{i}$ is equal to the sum of the interval times for all pallets in the system to pass the unloading module $m$ after releasing $P_{x_{i}}$. In mathematical expression

$$
\sum_{e=1}^{N_{p}} C_{y\left(x_{i}, e\right)}^{m}=S_{i}^{m}[k]-S_{i}^{1}[k]+T_{i}^{1}+L^{1} T_{c}: \quad i, x_{i} \in I
$$

where

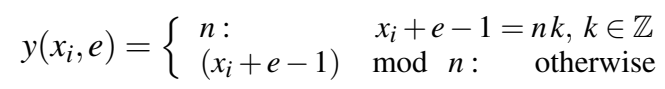

Defining $\hat{e}=N_{p}-e+1$, we have $g(i, \hat{e})=y\left(x_{i}, e\right)$ according to (12). This implies

$$
\sum_{e=1}^{N_{p}} C_{y\left(x_{i}, e\right)}^{m}=\sum_{e=1}^{N_{p}} C_{g\left(i, N_{p}-e+1\right)}^{m}=\sum_{\hat{e}=1}^{N_{p}} C_{g(i, \hat{e})}^{m}
$$

which gives (11) regarding (13).
For the variable $N_{p}$, the nonlinear relation 11 can be projected to the linear relations

$$
\begin{aligned}
& i \in I, \quad e \in I, \quad k \in K^{S} \\
& C N_{1}+r_{i}=S_{i}^{m}[k]-S_{i}^{1}[k]+T_{i}^{1}+L^{1} T_{C} \\
& r_{i}=\sum_{e=1}^{n} d_{i, e} \\
& C_{g(i, e)}^{m} \geq d_{i, e} \\
& d_{i, e} \geq C_{g(i, e)}^{m}-\left(1-x_{i, e}\right) C \\
& C x_{i, e} \geq d_{i, e} \\
& x_{i, e} \geq x_{i, e+1} \quad: \quad e \in I \backslash\{n\} \\
& N_{p}=N_{1} n+\sum_{e=1}^{n} x_{i, e}
\end{aligned}
$$

In (16), $r_{i}$ and $d_{i, e}$ are real variables, $N_{1}$ is an integer variable, and $x_{i, e}$ is a binary decision variable.

Buffer constraints: $S_{i}^{\ell}[k]-S_{i}^{\ell-1}[k]$ specifies the duration in which part $i$ stays in the segment $\ell$. According to (11), the constraint $\sum_{e=1}^{b^{\ell}} C_{g(i, e)}^{\ell}=S_{i}^{\ell}[k]-S_{i}^{\ell-1}[k]$ always demands $b^{\ell}$ number of pallets in the buffer. To let the number of pallets in the buffer not be higher than $b^{\ell}$, this constraint is modified to

$$
\begin{aligned}
& i \in I, \quad \ell \in M \backslash\{1\}, \quad k \in K^{s} \\
& \sum_{e=1}^{b^{\ell}} C_{g(i, e)}^{\ell} \geq S_{i}^{\ell}[k]-S_{i}^{\ell-1}[k]
\end{aligned}
$$

Variable constraints: For the design constraints, the set of variables with the following domains are defined .

$$
\begin{aligned}
& i \in I, \quad j \in I, \quad e \in I, \quad \ell \in M, k \in K \\
& N_{p}, N_{1}, b \in \mathbb{N} \\
& T_{i}^{\ell}, S_{i}^{\ell}[k], T_{c}, C_{i}^{\ell}, r_{i}, d_{i, e} \in \mathbb{R} \\
& \delta_{i, j}, x_{i, e} \in\{0,1\}
\end{aligned}
$$

Remark: Except the dynamic constraints, the other design constraints are valid when a pallet system is in the steady state phase. Hence, to reduce the number of the releasing time variables $S_{i}^{\ell}[k]$ in the constraints, we can select $k^{s}=1$, which results in elimination of transition states for the part releasing times in the design constraints. On the other hand, the number of the part set in a pallet system is always lower than $\left\lfloor N_{p} / n\right\rfloor+1$. Accordingly, the term $k^{s}+\left\lfloor N_{p} / n\right\rfloor$ may be considered as an upper bound value of $k_{U}^{s}$. When $N_{p}$ is variable, one estimated parameter larger than $N_{p}$ should be substituted in the term (for example, $k_{U}^{s}=\left\lfloor\frac{1}{n} \sum_{\ell=1}^{m} b^{\ell}\right\rfloor+1$ ).

\section{Optimal control}

The addressed mathematical models realize the control problem (3) as follows.

$$
\begin{aligned}
& F^{*}\left(N_{p}, v_{c}\right)=\min \quad F\left(N_{p}, T_{c}\right) \\
& \text { subject to } \\
& (7),(8),(9),(10),(16),(17),(18)
\end{aligned}
$$

Solving (19), the plant control set point $\left(N_{p}^{*}, T_{c}^{*}\right)$, which gives the desired cycle time and the minimal tension force, is obtained. 


\section{ROBUSTNESS ANALYSIS OF THE CONTROL DESIGN}

In practice machines are prone to failures, and operation times are not certainly deterministic. For a cyclic pallet system, these variations often lead to the deviation of the cycle time from the desired level. In this paper we are not going to introduce a framework for the stochastic analysis of the pallet system (this can be considered for future work). However, it is important to characterize the robustness of the control and the system design regarding the mentioned variations.

Let $W T=\sum_{i=1}^{n}\left(S_{i}^{m}[k]-S_{i}^{1}[k]\right)-\tau$ be the overall waiting times of a part set in the system buffers. According to (11), the optimal control set point $\left(N_{p}^{*}, T_{c}^{*}\right)$, the solution of (19), should satisfy the equation

$$
N_{p}^{*} C=\tau+n L T_{c}^{*}+W T
$$

Based on (20), an average variation on $\tau=\sum_{i=1}^{n} \sum_{\ell=1}^{m} \tau_{i}^{\ell}, \Delta \tau$, may impose the average deviation of the cycle time, $\Delta C$, and the waiting time, $\Delta W T(\Delta \tau)$. We say the control is robust if

$$
\frac{\Delta C}{C}=\frac{\Delta \tau+\Delta W T(\Delta \tau)}{\tau+n L T_{c}^{*}+W T}
$$

is in an acceptable rage. However, $W T$ and $\Delta W T(\Delta \tau)$ are not defined as explicit functions of the system parameters, while $\Delta C$ can simply be measured during the production phase. Hence, we characterize a robust control such that with the minimum changes in its set point $\left(N_{p}^{*}, T_{c}^{*}\right)$, the term $\Delta C$ can be eliminated or significantly be reduced in the running phase of the pallet system.

Over production with $\Delta C<0$ is not problematic because a cancelation of $\Delta C$ can then be achieved by increasing $T_{c}^{*}$ or decreasing $N_{p}^{*}$, resulting in the force reduction according to (6). The dilemma occurs when a trade off between the rise of the force value and the reduction of $\Delta C>0$ should be made. Keeping the discrete parameter $N_{p}^{*}$ unchanged, this leads to the reduction $T_{c}^{*}-\left|\Delta T_{c}\right|$, where $\Delta T_{c}<0$ and

$$
n L\left|\Delta T_{c}\right|=\Delta \tau+\Delta W T(\Delta \tau)+\Delta W T\left(\Delta T_{c}\right)
$$

and $\Delta W T\left(\Delta T_{c}\right) \geq 0$ is a $W T$ deviation due to this reduction (regarding (20), $W T$ is a decreasing function of $T_{c}$ for given $N_{p}$ and $C$ ). On the other hand, (20) and (21) imply that $\Delta \tau+\Delta W T(\Delta \tau)=N_{p}^{*} C \frac{\Delta C}{C}$. Therefore, the condition

$$
\left|\Delta T_{c}\right| \geq \frac{\varepsilon N_{p} C}{n L}
$$

realizes a criterion for the robust control to cancel the aimed error $\varepsilon>\frac{\Delta C}{C}$.

To verify the optimal control robustness based on (23), we develop the concept of the worst but safe control that is defined as follows.

$$
\begin{aligned}
& F^{w s}\left(N_{p}, T_{c}\right)=\max F\left(N_{p}, T_{c}\right) \\
& \text { subject to } \\
& (7),(8),(9),(10),(16),(17),(18)
\end{aligned}
$$

Denoting $\left.F^{w s}\left(T_{c}\right)\right|_{N_{p}^{*}}=F^{w s}\left(N_{p}^{*}, T_{c}\right)$, which means that (24) is parameterized by $N_{p}=N_{p}^{*}$, and substituting (23) in (6), the constraint

$$
\left.\Delta F^{w s}\right|_{N_{p}^{*}}=\left.F^{w s}\left(T_{c}\right)\right|_{N_{p}^{*}}-\left.F^{*}\left(T_{c}\right)\right|_{N_{p}^{*}} \geq \varepsilon g m_{p} \mu_{c p} N_{p}^{*}
$$

is a necessary condition for $\left(N_{p}^{*}, T_{c}^{*}-\frac{\varepsilon N_{p}^{*} C}{n L}\right)$ to meet the error cancelation. If (25) is not held, then $\left(N_{p}^{*}, T_{c}^{*}\right)$ is not robust and we should search for $\left|\Delta N_{p}\right|$ by which both (19) and (25) are realized for $N_{p}=N_{p}^{*}+\Delta N_{p}$.

\section{ILLUSTRATIVE EXAMPLES}

In this section we apply the optimal control to 8 case studies. To do so, first we consider two cyclic pallet system benchmarks based on X85 technology. One has 6 modules and 4 part types $(\mathrm{A}, . ., \mathrm{D})$, and the other includes 20 modules and 9 part types $(\mathrm{A}, \ldots, \mathrm{I})$. These benchmarks present small and big size of cyclic pallet systems in practice. The related data for the X85 pallet system are shown in Table I.

TABLE I

DATA FOR X85 WITH $\mu_{c p}=0.3, \mu_{c s}=0.1$, AND $m_{P}=10[\mathrm{KG}]$

\begin{tabular}{cccccccc}
\hline \hline & & & & & & & \\
SYS & $m_{c}\left[\frac{\mathrm{kg}}{\mathrm{m}}\right]$ & $\widetilde{V}\left[\frac{\mathrm{m}}{\mathrm{s}}\right]$ & $\widetilde{F} 1[\mathrm{~N}]$ & $\widetilde{F} 2[\mathrm{~N}]$ & $T$ & $\lambda$ & $\widetilde{I}\left[\frac{\mathrm{kgm}}{\mathrm{s}}\right]$ \\
\hline Small & 1.5 & 0.5 & 1250 & 800 & 12 & 35 & 3.33 \\
Big & 2 & 0.5 & 1250 & 1250 & 12 & 26 & 3.33
\end{tabular}

Furthermore, we randomly generate system data for the cases as follows.

$$
\begin{aligned}
& I=\{1,2, \ldots, n\}, M=\{1,2, \ldots, m\}, M=\{2, \ldots, m-1\} \\
& 0 \leq p_{i}^{\ell} \leq 80[\mathrm{~s}]: i \in I, \ell \in M \\
& \tau_{i}^{\ell}=\max \left\{0, p_{i}^{\ell}-20\right\}[\mathrm{s}]: i \in I, \ell \in M \\
& 5 \leq \tau_{i}^{\ell}=\tau_{j}^{\ell} \leq 12[\mathrm{~s}]: i, j \in I, \ell \in\{1, m\} \\
& \max \left\{\sum_{i=1}^{n} \tau_{i}^{\ell}: \ell \in M\right\} \leq C \leq 1.2 \max \left\{\sum_{i=1}^{n} \tau_{i}^{\ell}: \ell \in M\right\}[\mathrm{s}] \\
& 2 \leq b^{\ell} \leq 4 \\
& L^{\ell}=0.5 b^{\ell}+0.1[\mathrm{~m}]: \ell \in M \backslash\{1\} \\
& L^{1}=0.5 b^{1}+1.1[\mathrm{~m}]
\end{aligned}
$$

The cases 1 to 4 are related to the small benchmark, and the cases 5 to 8 are considered for the big benchmark. For example, Table II depicts the data for Case 1, and Table III includes the data regarding Case 5.

TABLE II

SySTEM DATA FOR CASE 1 WITH $C=142$ AND SCHEDULE DBAC

\begin{tabular}{ccccccc}
\hline \hline$M^{\ell}$ & $T_{A}$ & $T_{B}$ & $T_{C}$ & $T_{D}$ & $L^{\ell}$ & $b^{\ell}$ \\
& {$[\mathrm{s}]$} & {$[\mathrm{s}]$} & {$[\mathrm{s}]$} & {$[\mathrm{s}]$} & {$[\mathrm{m}]$} & \\
\hline$M^{1}$ & 5 & 5 & 5 & 5 & 2.6 & 3 \\
$M^{2}$ & 17 & 53 & 47 & 11 & 1.6 & 3 \\
$M^{3}$ & 40 & 0 & 0 & 44 & 1.1 & 2 \\
$M^{4}$ & 24 & 51 & 0 & 25 & 1.1 & 2 \\
$M^{5}$ & 41 & 0 & 0 & 54 & 1.1 & 2 \\
$M^{6}$ & 7 & 7 & 7 & 7 & 2.1 & 4 \\
\hline
\end{tabular}

We solve the optimization model (19) for the cases, using GLPK software, [31]. The derived minimal tension force $F$, the upper bound force $F^{u}$, the optimal control parameters, and the computation time $C T$ are illustrated in Table IV. 
TABLE III

SySTEM DATA FOR CASE 5 WITH $C=370$ AND SCHEDULE AGFCDEIHB

\begin{tabular}{cccccccccccc}
\hline \hline$M^{\ell}$ & $T_{A}^{\ell}$ & $T_{B}^{\ell}$ & $T_{C}^{\ell}$ & $T_{D}^{\ell}$ & $T_{E}^{\ell}$ & $T_{F}^{\ell}$ & $T_{G}^{\ell}$ & $T_{H}^{\ell}$ & $T_{I}^{\ell}$ & $L^{\ell}$ & $b \ell$ \\
& {$[\mathrm{s}]$} & {$[\mathrm{s}]$} & {$[\mathrm{s}]$} & {$[\mathrm{s}]$} & {$[\mathrm{s}]$} & {$[\mathrm{s}]$} & {$[\mathrm{s}]$} & {$[\mathrm{s}]$} & {$[\mathrm{s}]$} & {$[\mathrm{m}]$} & \\
\hline$M^{1}$ & 5 & 5 & 5 & 5 & 5 & 5 & 5 & 5 & 5 & 2.1 & 2 \\
$M^{2}$ & 0 & 56 & 31 & 22 & 35 & 12 & 32 & 0 & 0 & 2.1 & 4 \\
$M^{3}$ & 0 & 31 & 34 & 31 & 52 & 46 & 57 & 0 & 47 & 1.1 & 2 \\
$M^{4}$ & 17 & 0 & 0 & 23 & 0 & 11 & 13 & 0 & 0 & 2.1 & 4 \\
$M^{5}$ & 49 & 38 & 0 & 29 & 0 & 33 & 53 & 53 & 0 & 1.1 & 2 \\
$M^{6}$ & 0 & 12 & 0 & 0 & 0 & 22 & 0 & 58 & 0 & 1.1 & 2 \\
$M^{7}$ & 55 & 42 & 21 & 31 & 27 & 0 & 24 & 60 & 56 & 1.1 & 2 \\
$M^{8}$ & 58 & 60 & 13 & 0 & 47 & 48 & 0 & 14 & 37 & 1.1 & 2 \\
$M^{9}$ & 16 & 0 & 0 & 21 & 11 & 30 & 12 & 0 & 17 & 2.1 & 4 \\
$M^{10}$ & 59 & 55 & 25 & 28 & 245 & 0 & 0 & 0 & 50 & 2.1 & 4 \\
$M^{11}$ & 0 & 39 & 0 & 22 & 21 & 0 & 32 & 42 & 37 & 1.1 & 2 \\
$M^{12}$ & 43 & 0 & 55 & 48 & 29 & 0 & 36 & 40 & 33 & 1.6 & 3 \\
$M^{13}$ & 31 & 0 & 56 & 60 & 59 & 29 & 14 & 0 & 21 & 1.1 & 2 \\
$M^{14}$ & 33 & 55 & 31 & 58 & 38 & 0 & 0 & 20 & 57 & 2.1 & 4 \\
$M^{15}$ & 24 & 24 & 0 & 42 & 58 & 24 & 0 & 0 & 48 & 1.1 & 2 \\
$M^{16}$ & 0 & 52 & 53 & 0 & 0 & 41 & 0 & 36 & 0 & 1.6 & 3 \\
$M^{17}$ & 0 & 45 & 38 & 0 & 53 & 57 & 0 & 45 & 0 & 1.1 & 2 \\
$M^{18}$ & 34 & 0 & 0 & 0 & 0 & 43 & 0 & 50 & 24 & 1.6 & 3 \\
$M^{19}$ & 46 & 44 & 60 & 30 & 0 & 0 & 58 & 45 & 31 & 1.1 & 2 \\
$M^{20}$ & 5 & 5 & 5 & 5 & 5 & 5 & 5 & 5 & 5 & 1.1 & 2 \\
\hline
\end{tabular}

The results show that the optimal control in average puts a small force on the drive unit and significantly limits the maximum tension force.

TABLE IV

THE OPTIMAL CONTROL SCENARIO FOR CASES 1 TO 8

\begin{tabular}{cccccc}
\hline \hline & & & & & \\
CASE & $N_{p}$ & $T_{c}\left[\frac{\mathrm{s}}{\mathrm{m}}\right]$ & $F[\mathrm{~N}]$ & $F^{u}[\mathrm{~N}]$ & $\mathrm{CT}[\mathrm{s}]$ \\
\hline 1 & 6 & 8.54 & 64.5 & 254.4 & 1.5 \\
2 & 6 & 9.78 & 60 & 253.7 & 0.6 \\
3 & 5 & 4.3 & 64.3 & 220.4 & 0.2 \\
4 & 6 & 6.8 & 72.5 & 256 & 0.2 \\
5 & 21 & 4.34 & 363.1 & 899 & 8.4 \\
6 & 21 & 4.52 & 343.2 & 897 & 20 \\
7 & 17 & 2.25 & 245.2 & 743 & 15 \\
8 & 16 & 2.7 & 215 & 694 & 25
\end{tabular}

For the robust analysis, we only consider Cases 1 and 5 . Regarding Case 1, Fig. 4 depicts the optimal control and worst but safe control for $N_{p} \in[5,14]$. The vertical solid (black) lines in this figure determine the range of forces on the drive unit, satisfying the safety conditions and the cycle time $C=142[\mathrm{~s}$. It is interesting to see that the domain of safe control policies corresponds to a wide range of forces $(64.5[\mathrm{~N}] \leq F \leq 333.1[\mathrm{~N}])$. This gives more credits to the optimal control which provides the minimal force $64.5[\mathrm{~N}]$ for the set point $\left(N_{p}^{*}=6, T_{c}^{*}=8.54\right)$. Setting this optimal point, the error with magnitude $\varepsilon=0.2$ can be canceled by only changing $T_{c}$. This implies that the optimal control is robust for the average cycle time deviation $\Delta C \leq \varepsilon C=28.4[\mathrm{~s}]$. Rising $N_{p}$ and following the optimal control path (the light green line), the robustness of the control increases until the set point $\left(N_{p}=10, T_{c}=20\right)$, which provides the maximum robustness $(\varepsilon=0.4)$, although it does not sustain the minimal force $(F=124)$.

Another, interesting point in the figure is $\left(N_{p}=5, T_{c}=\right.$ 2.19). This point presents the minimal work in process, proposed by the lean philosophy as the optimal set point. Although the force in this point is very close to the minimal force, this control set point is not robust and not advised for the system. Only the small deviation $\Delta C \geq 1.42[\mathrm{~s}]$ can violate the desired production rate.

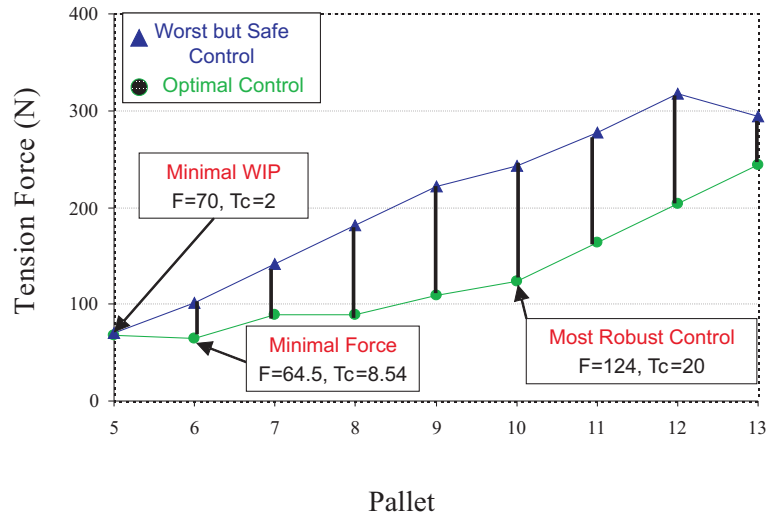

Fig. 4. Robustness analysis for Case 1

A similar robust analysis can be applied for Case 5 . This results in Fig. 5. Assume that the request is to cancel error with $\varepsilon=0.15$. Based on this assumption, the optimal control set point $\left(N_{p}=5, T_{c}=4.34\right)$ is not robust enough $(\varepsilon=0.083)$. Interestingly, adding one more pallet decreases the control robustness. As a matter of fact, the required error cancelation can only be obtained for $N_{p}=24$. Consequently, the optimal control is set for $\left(N_{p}=24, T_{c}=8.46\right)$.

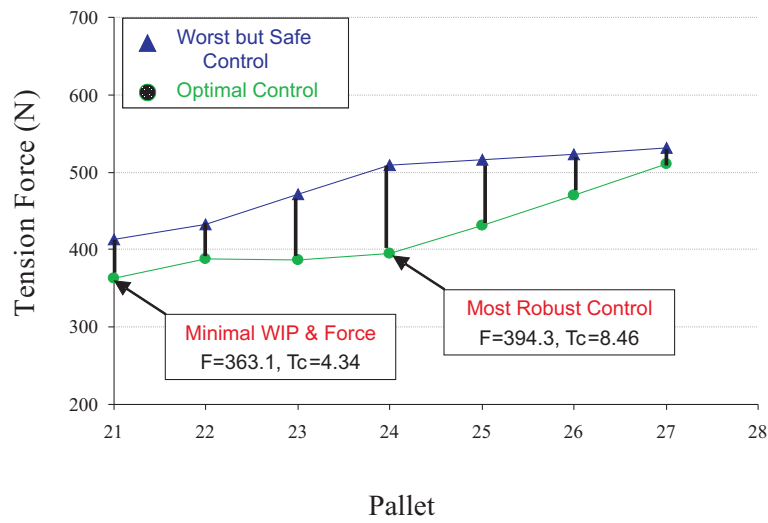

Fig. 5. Robustness analysis for Case 5

\section{CONCLUSION AND FUTURE WORKS}

This paper provided a formal tool to design and characterize a sustainable and robust control for cyclic pallet systems. The designed control is optimal such that it minimizes the force load on the drive unit, satisfies the safety conditions on the system resources, and fulfills a desired production rate. This tool was applied to some case studies based on small and big one-loop pallet systems. The achieved results showed that a significant amount of the force was reduced, while safely running the systems to meet their desired cycle times. Furthermore, the domain of set points realizing a robust control was derived for one small and one big case. 
This formal tool was developed according to the assumptions denoted in Section II. One-loop pallet system, chain based drive unit, and the horizontal movement are those which can be relaxed with minimal impact on the tool framework. This tool can be applied to a multiple-loop pallet system, loop by loop with assuming a constant work in process (WIP) in each loop. For roller based drive units and a slope for the conveyor line, only the constant coefficients in (6) should be modified. However, the other assumptions are essential to define a classical deterministic permutation flow shop, [32]. Introduction of a variable schedule dramatically increases the model computation time due to the binary decision variables $\delta_{i, j}$. Dealing with this time complexity, a heuristic approach based on the FIFO policy has been developed in [33]. Still, a continuation of this work can be considered for a general queuing policy.

\section{REFERENCES}

[1] T. Yamada, S. Kataoka, "On some LP problems for performance evaluation of timed marked graphs", IEEE Transactions on Automatic Control, Vol.39, pp. 696-698, 1994.

[2] R.L. Rardin, "Optimization in operations research", Prentice Hall, 1998.

[3] H.T. Papadopoulos and C. Heavey, "Queueing theory in manufacturing systems analysis and design: A classification of models for production and transfer lines", European Journal of Operational Research, Vol. 92, No. 1, pp. 1-27, 1996.

[4] H. Tempelmeier, H. Kuhn, "Flexible manufacturing systems: Decision support for design and operation", Wiley Series in Systems Engineering, 1993.

[5] S.B Gershwin and L.M. Werner, "An approximate analytical method for evaluating the performance of closed-loop flow systems with unreliable machines and finite buffers", International Journal of Production Research, Vol. 45, pp. 30853111, 2007.

[6] J. Li, D.E. Blumenfeld, N. Huang, and J.M. Alden, "Throughput analysis of production systems: recent advances and future topics", International Journal of Production Research, Vol. 47, No. 14, pp. 3823-3851, 2009.

[7] J. Li and S.M. Meerkov. Production system eneginerring. Springer, New Yoek, 2009.

[8] C.G. Cassandras and S. Lafortune, "Introduction to discrete event systems", Springer, 2nd edition, 1999.

[9] T. Murata, "Petri Nets: Properties, analysis and applications", Proc. of the IEEE, Vol. 77, No.4, pp. 541-580, 1989.

[10] C.V. Ramamoorthy, G.S. Ho, "Performance evaluation of asynchronous concurrent systems using Petri nets", IEEE Transactions on Software Engineering, Vol.6, pp. 440-449, 1980.

[11] J. Magott, ’Performance evaluation of concurrent systems using Petri nets", Information Processing Letters, vol. 18, No. 1, pp. 713, 1984.

[12] S. Bogdan, F.L. Lewis, Z. Kovacic, and J. Mireles Jr. " Manufacturing systems control design: a matrix-based approach", Advances in Industrial Control, Springer, 2006.

[13] S. Biller, S.P. Marin, S.M. Meerkov, and L. Zhang, "Closed Bernoulli production Lines: Analysis, Continuous Improvement, and Leanness", IEEE Transactions on Automation Science and Engineering, Vol. 6, No. 1, pp. 168-180, 2009.

[14] M.S Han, D.J Park" Performance analysis and optimization of cyclic production line", IIE Transactions, Vol 34, pp. 411-422, 2002.

[15] Y. Frein, C. Commault, and Y. Dallery, "Modeling and analysis of closed-loop production lines with unreliable machines and finite buffers", IIE Transactions, Vol 28, pp. 545-554, 1996.

[16] B. Pourbabai, " Performance modeling of a closed loop material handling system", European Journal of Operational Research , Vol 32, pp 340-352, 1987.

[17] S.P. Sethi, C. Sriskandarajah, S.L. van de Velde, M.Y. Wang, and H. Hoogeveen, "Minimizing makespan in a pallet-constrained flowshop", Journal of Scheduling, Vol. 2, pp. 115-133, 1999.

[18] M.Y. Wang, S.P. Sethi, C. Sriskandarajah, and S.L. van de Velde, "Minimizing makespan in a flowshop with pallet requirements", Inform. Systems Oper. Res. INFOR, nr. 35, pp. 277-285, 1997.
[19] C. Chu, J.M. Proth, and S.P Sethi, "Heuristic procedures for minimizing makespan and the number of required pallets ", European Journal of Operational Research, Vol. 86, pp. 491-502, 1995.

[20] O. Korbaa, H. Camus, and J.C Gentina "A new cyclic scheduling algorithm for flexible manufacturing systems", The International Journal of Flexible Manufacturing Systems, Vol 14, pp. 173-187, 2002.

[21] F. Chauvet, J.W. Herrmann, and J.M. Proth, Optimization of cyclic production systems: a heuristic approach, IEEE Transactions on Robotics and Automation, Vol 19, No. 1, pp. 150-154, 2003.

[22] T. Hsu, O. Korbaa b, R. Dupas, and G. Goncalves, "Cyclic scheduling for F.M.S.: Modelling and evolutionary solving approach", European Journal of Operational Research, Vol. 191, pp. 464-484, 2008.

[23] M. Mashaei, B. Lennartson, F. Sannehed, and G. Abbestam, "Optimal design of a decoupled multiple-loop pallet system for flexible cyclic manufacturing plants", CASE, IEEE Conference on Automation Science and Engineering, pp. 433-438, 2010.

[24] P. Solot, "A heuristic method to determine the number of pallets in a flexible manufacturing system with several pallet types", The International Journal of Flexible Manufacturing Systems, Vol.2, pp. 191-216, 1990.

[25] M.P. Groover, "Automation, production systems, and computerintegrated manufacturing", Second Edition, Prentice Hall International 2001.

[26] K. Ramani, D. Ramanujan, W.Z. Bernstein, F. Zhao, J. Sutherland, C. Handwerker, J.K. Choi, H. Kim, D. Thurston, "Integerated sustainable life cycle design: A review", Journal of Mechanical Design, Vol.132, pp. 091004-1-091004-15, 2010.

[27] J.D. Little, "A proof of the queueing formula $L=\lambda W$ ", Operation Research, Vol. 9, No. 3, pp. 383-387, 1961.

[28] W.J. Hopp, and M.L. Spearman, "Factory physics: Foundations of manufacturing management", 2nd edition, IrwinlMcGraw Hill, NY, 2000.

[29] http://www.flexlink.com.

[30] M. Mashaei, B. Lennartson, and G. Abbestam, "Green and Lean Control of Cyclic Pallet Systems", 7th Annual IEEE Conference on Automation Science and Engineering, pp. 642-647, 2011.

[31] http://www.gnu.org/software/glpk, 2008.

[32] M. L. Pinedo, "Scheduling: Theory, algorithms, and systems", Third Edition, Springer, 2008.

[33] M. Mashaei, B. Lennartson, "Sustainable and robust Control of Cyclic Pallet Systems", submitted to IEEE Transactions on Automation Science and Engineering, 2012. 